\title{
MORTALIDADE POR CAUSAS EXTERNAS EM IDOSOS EM MINAS GERAIS, BRASIL
}

\author{
Mortality from external causes among elderly in Minas Gerais, Brazil \\ Mortalidad por causas externas en ancianos en Minas Gerais, Brasil
}

Ludmila Mourão Xavier Gomes

Thiago Luis de Andrade Barbosa ${ }^{2}$

Antonio Prates Caldeira ${ }^{3}$

\section{RESUMO}

perfil dos óbitos dessa faixa etária, em Minas Gerais, Brasil, no período de 1999 a 2008. Os dados foram obtidos a partir do Sistema de Informações sobre Mortalidade do Ministério da Saúde e do Departamento de Informática do Ministério da Saúde. Os resultados apontaram coeficientes crescentes de mortalidade em idosos durante os anos estudados. Destacaramse como principais causas as quedas e os acidentes de transporte. Também foram registradas taxas ascendentes dos casos de homicídios e suicídios, especialmente em idosos do sexo masculino. São necessárias medidas preventivas imediatas, pois os idosos se mostram cada vez mais sujeitos às mortes por causas externas.

Palavras-chave: Enfermagem Geriátrica. Idoso. Mortalidade. Causas Externas. Sistemas de Informação.

\begin{abstract}
This is a descriptive study that aimed to analyze the mortality among elderly persons by external causes and the death profile of this age in Minas Gerais, Brazil, from 1999 to 2008. The data were obtained from the Mortality Information System and from the Department of Informatics of the Ministry of Health. The results showed increasing mortality coefficients in elderly people during the years studied. The falls and transport accidents stood out as major causes of deaths. The results also showed increasing rates of homicides and suicides, especially in elderly males. Immediate preventive measures are necessary, since the elderly people are becoming increasingly subject to deaths from external causes.
\end{abstract}

Keywords: Geriatric Nursing. Aged. Mortality. External Causes. Information Systems

\section{Resumen}

Este es un estudio descriptivo con el objetivo de examinar la mortalidad por causas externas en los ancianos y el perfil de los óbitos en este grupo de edad, en Minas Gerais, Brasil, desde 1999 hasta 2008. Los datos fueron obtenidos del Sistema de Informaciones sobre Mortalidad y del Departamento de Informática del Ministerio de Salud. Los resultados mostraron aumento de los coeficientes de la mortalidad en ancianos durante los años estudiados. Las caídas y accidentes de transporte se destacaron como las principales causas de muertes. Los resultados también mostraran crecientes tasas de homicidios y suicidios, especialmente en los ancianos varones. Medidas inmediatas de prevención son necesarias, ya que los ancianos son cada vez más sujetos a las muertes por causas externas.

Palabras clave: Enfermería Geriátrica. Anciano. Mortalidad. Causas Externas. Sistemas de Información.

${ }^{1}$ Enfermeira, Mestranda em Ciências da Saúde pela Universidade Estadual de Montes Claros, Unimontes. Montes Claros- MG. Brasil. E-mail: ludyxavier@yahoo.com.br, ${ }^{2}$ Enfermeiro, Coordenador de Epidemiologia pela Secretaria Municipal de Januária. Januária - MG. Brasil. E-mail: tl_andrade@yahoo.com.br, ${ }^{3}$ Médico, Doutor, Professor do curso de Pós-Graduação (Mestrado) em Ciências da Saúde da Universidade Estadual de Montes Claros. Montes Claros-MG. Brasil. E-mail: antonio.caldeira@uninontes.br 


\section{INTRODUÇÃO}

As causas externas ganharam destaque nos estudos de mortalidade nas últimas décadas em decorrência da intensificação das mortes por homicídios a partir dos anos 80, que chegaram a alcançar, em 2004, 39,1\% do total de mortes por causas externas no país., ${ }^{1,2}$ Analisar e compreender a distribuição da mortalidade por causas externas nos diferentes estratos etários da população é essencial para proposição de medidas que possam antecipar situações de risco.

Os acidentes e violências estão entre as principais causas de morte na população jovem e adulta. Entretanto, estudos vêm revelando que essas causas também são merecedoras de atenção entre as pessoas idosas. Pesquisas desenvolvidas sobre a situação da violência, especificamente no trânsito demonstraram que, embora as mortes por essas causas estejam concentradas em adultos jovens, as taxas de mortalidade apresentam-se crescentes à medida que avança a idade. ${ }^{3,4}$

Considerado um país de jovens, o Brasil chega ao século XXI com uma perspectiva de envelhecimento populacional, que deve situá-lo entre as nações do mundo com o maior número de idosos. Isso significa muito mais do que apenas indicadores estatísticos, pois resulta em implicações profundas, políticas, sociais e no sistema de saúde. ${ }^{4}$ Os dados do Censo 2000 mostraram o envelhecimento da população brasileira, o que é considerado pela demografia como um sinal de desenvolvimento. Contudo, o aumento da ocorrência de determinados grupos de agravos, entre os quais as causas externas, como os acidentes e violências, deve ser objeto de preocupação e discussão entre os profissionais da saúde. ${ }^{5,6}$

No Brasil, a população idosa não costuma ser prioridade nos estudos sobre mortalidade ocasionada por causas externas. ${ }^{5}$ Existe um predomínio desse grupo de causas entre os jovens, que exibem altos coeficientes e grande número de casos e, indiscutivelmente, devem ser objeto de políticas públicas especialmente voltadas para o enfrentamento do problema. Por outro lado, se os números absolutos dos óbitos por causas externas entre os idosos não chamam atenção, o mesmo não pode ser afirmado em relação aos coeficientes. ${ }^{5}$ Estudos realizados em São Paulo (SP), no ano de 1999, e no país, em 2000, registraram coeficientes de mortalidade por causas externas em idosos na ordem de 184,3/100 mil e 92,1/100 mil, respectivamente. ${ }^{5,7}$ Coeficientes estes superiores a algumas faixas etárias entre adultos em ambos estudos.

A elevada vulnerabilidade fisiológica dos idosos deve contribuir para uma maior mortalidade, devido a uma combinação de fatores que inclui dificuldades nos campos da percepção e equilíbrio, fragilidade do sistema musculoesquelético, diminuição da capacidade visual, entre outras $^{4}$. Porém, isso não significa que traumas e lesões sejam consequência inevitável da idade, pois a premissa fundamental da saúde pública de que as causas externas podem ser previsíveis e, portanto, evitáveis, vale para todas as idades. ${ }^{8}$ Entre as causas externas, os óbitos relacionados aos acidentes de transporte são os mais comuns. Deve-se considerar, todavia, que existe um crescimento das mortes violentas, provavelmente, reflexo dos problemas urbanos contemporâneos, o que tem motivado estudos, com importância crescente no cenário nacional e internacional., 910

Nesse sentido, descrever características da população idosa, como o perfil de mortalidade e os seus aspectos mais importantes, é tarefa imprescindível. Para cada região, é fundamental que as condições de saúde, adoecimento e morte da população sejam bem conhecidas e subsidiem ações de proteção à vida. ${ }^{4} \mathrm{~A}$ partir desses pressupostos e do reconhecimento da escassa literatura sobre o tema, o presente estudo teve por objetivo analisar a mortalidade por causas externas em idosos, traçando o perfil dos óbitos dessa faixa etária, em Minas Gerais, no período de 1999 a 2008.

\section{MÉTODO}

Trata-se de um estudo descritivo de série temporal baseado em dados secundários de banco de dados oficiais. Para a definição da população idosa, optou-se por estudar a faixa etária de 60 anos ou mais, idade limite ou inicial do envelhecimento, conforme estabelecida pela Política Nacional do ldoso. ${ }^{11}$

Os óbitos dos idosos residentes em Minas Gerais no período de 1999 a 2008 foram obtidos do Sistema de Informações sobre Mortalidade (SIM) do Ministério da Saúde. As informações relativas à população de idosos residentes no estado de Minas Gerais nos 10 anos estudados foram obtidas do Departamento de Informática do SUS (Datasus). ${ }^{12}$ As causas básicas de óbito foram analisadas por sexo e idade e estudadas segundo os agrupamentos da Classificação Internacional de Doenças, $10^{a}$ Revisão (CID-10). ${ }^{13}$ Os óbitos por causas externas em idosos foram analisados por meio de coeficientes específicos de mortalidade.

Foram realizadas algumas análises complementares de informações sobre a população geral (de referência) com o objetivo de se estabelecer comparações com a faixa etária de idosos.

\section{RESULTADOS E DISCUSSÃO}

No período estudado, o percentual de óbitos em idosos passou de $55,8 \%$ no ano de 1999 para $61,7 \%$ em 2008, entre todos os óbitos observados. Particularmente em relação às causas externas, objeto desse estudo, observou-se também um crescimento do percentual de óbitos em pessoas com 60 anos ou mais. Esse percentual era de 15,8\% em 1999 e passou para 17,4\% em 2008. Esses números destacam a relevância de estudos na área e corroboram as implicações políticas, sociais e sobre o próprio sistema de saúde decorrentes do processo de 
envelhecimento populacional. Os resultados apresentados a seguir retratam o universo dos óbitos por causas externas ocorridos em idosos residentes em Minas Gerais, no período de 1999 a 2008. Contudo, é importante salientar que o estudo se deteve, principalmente, às observações no período de 2000 a 2007 devido aos dados relevantes encontrados neste intervalo.
Previamente a década de 60, as violências situavam-se no quarto lugar no perfil de mortalidade geral, posteriormente passando para o segundo lugar nos anos 80 e 90.8 Tendo a velhice como uma fase do desenvolvimento humano cada vez mais evidente e como oportunidade social de trazer novas perspectivas para a população em geral, alguns autores ressaltam a importância de estudos de mortalidade na população idosa. ${ }^{4}$

Tabela 1: Número de óbitos e coeficientes de mortalidade por causas externas em idosos (por 100.000 hab), segundo faixa etária, em Minas Gerais, no período de 1999 a 2008.

\begin{tabular}{|c|c|c|c|c|c|c|c|c|}
\hline \multirow{3}{*}{ Ano } & \multicolumn{8}{|c|}{ Faixa Etária } \\
\hline & \multicolumn{2}{|c|}{60 a 69 anos } & \multicolumn{2}{|c|}{70 a 79 anos } & \multicolumn{2}{|c|}{80 anos e mais } & \multicolumn{2}{|c|}{ Total } \\
\hline & $\mathrm{N}$ & Coef. & $\mathrm{N}$ & Coef. & $\mathrm{N}$ & Coef. & $\mathrm{N}$ & Coef. \\
\hline 1999 & 587 & 69,45 & 413 & 97,34 & 371 & 220,57 & 1371 & 95,36 \\
\hline 2000 & 455 & 49,16 & 363 & 73,01 & 273 & 135,03 & 1091 & 67,14 \\
\hline 2001 & 538 & 57,48 & 410 & 81,59 & 363 & 177,75 & 1311 & 79,80 \\
\hline 2002 & 562 & 59,40 & 405 & 79,76 & 360 & 174,52 & 1327 & 79,93 \\
\hline 2003 & 619 & 64,78 & 487 & 94,99 & 445 & 213,71 & 1551 & 92,51 \\
\hline 2004 & 643 & 66,63 & 521 & 100,66 & 394 & 187,52 & 1558 & 92,04 \\
\hline 2005 & 667 & 67,61 & 519 & 98,16 & 457 & 213,11 & 1643 & 94,99 \\
\hline 2006 & 678 & 67,97 & 611 & 114,34 & 568 & 262,20 & 1857 & 106,20 \\
\hline 2007 & 737 & 66,23 & 604 & 91,88 & 639 & 216,33 & 1980 & 95,86 \\
\hline 2008 & 680 & 59,22 & 648 & 95,28 & 724 & 231,91 & 2052 & 95,86 \\
\hline
\end{tabular}

Fonte: Departamento de Informática do SUS (DATASUS), 1999 a 2008.

Os coeficientes de mortalidade em idosos por causas externas são apresentados na Tabela 1, com distribuição entre os três grupos etários de idosos. Registra-se, para todas as faixas etárias estudadas, um importante declínio entre os anos de 1999 e 2000 e, nos anos posteriores, um crescimento significativo até 0 ano de 2006, quando os cálculos novamente registram um declínio do coeficiente de mortalidade por causas externas em idosos para o ano de 2007. É importante salientar que os anos de declínios acentuados (2000 e 2007) coincidem com ajustes das estimativas populacionais, pois representam os anos do Censo Demográfico (2000) e da Contagem
Populacional (2007). Assim, esses anos devem ser analisados de forma diferenciada e comparativa entre si, e não no contexto da série histórica. Registra-se, então, um nítido aumento entre os anos de 2000 e 2007 e aumento também nos anos em que 0 denominador do cálcul o foi aferido a partir de estimativas populacionais (2001 a 2006). Ou seja, ao se comparar os coeficientes de mortalidade por causas externas para os anos intercensitários, observou-setambém um aumento dos mesmos em todas as faixas etárias. Cabe destacar dessa análise que o grupo etário acima de 80 anos apresentou os maiores coeficientes, o que retrata maior risco de mortes por causas externas para essa população.

Tabela 2: Número de óbitos e coeficientes de mortalidade por causas externas em idosos (por 100.000 hab), segundo sexo, em Minas Gerais, no período de 1999-2008.

\begin{tabular}{rrrrrrr}
\hline \multirow{2}{*}{ Ano } & \multicolumn{2}{c}{ Masculino } & \multicolumn{2}{c}{ Feminino } & \multicolumn{2}{c}{ Total } \\
\cline { 2 - 7 } 1999 & $\mathbf{N}$ & \multicolumn{1}{c}{ Coef. } & N & Coef. & N & \multicolumn{1}{c}{ Coef. } \\
\hline 2000 & 710 & 134,42 & 492 & 62,77 & 1371 & 95,36 \\
2001 & 872 & 96,75 & 381 & 42,76 & 1091 & 67,14 \\
2002 & 863 & 117,56 & 439 & 48,72 & 1311 & 79,80 \\
2003 & 1027 & 135,15 & 464 & 50,95 & 1327 & 79,93 \\
2004 & 1006 & 131,69 & 552 & 56,97 & 1551 & 92,51 \\
2005 & 1067 & 136,75 & 576 & 69,43 & 1558 & 92,04 \\
2006 & 1116 & 141,52 & 691 & 71,98 & 1857 & 94,99 \\
2007 & 1262 & 135,45 & 718 & 63,33 & 1980 & 95,20 \\
2008 & 1250 & 129,35 & 802 & 68,30 & 2052 & 95,86 \\
\hline
\end{tabular}

Fonte: Departamento de Informática do SUS (DATASUS), 1999 a 2008. 
A distribuição do coeficiente de mortalidade por causas externas também registrou crescimento para ambos os sexos, variando de $96,75 / 100$ mil a $135,45 / 100$ mil para o sexo masculino, entre os anos de 2000 e 2007, e de 42,76/100 mil a 63,33/100mil, para o sexo feminino no mesmo período (Tabela 2). De forma similar, os anos intercensitários também registraram crescimento dos coeficientes. A razão entre os coeficientes masculino/feminino variou de 1,9 a 2,4 ao longo da série estudada, o que difere do estudo realizado no Brasil em 2001 no qual a razão de sexos entre os coeficientes de mortalidade por causas externas foi igual a $5,5 .{ }^{5} \mathrm{~A}$ diferença observada revela um padrão de mortalidade diferencial entre os sexos, em relação ao restante do país. Contudo, as razões para essas diferenças não foram encontradas na literatura e demandam a realização de novas análises e estudos específicos para esclarecimento do fato.

Tabela 3 - Percentuais e coeficientes e de mortalidade por causas externas em idosos (por 100.000 hab) segundo os principais grupos de causas em Minas Gerais, no período de 1999-2008.

\begin{tabular}{|c|c|c|c|c|c|c|c|c|c|c|}
\hline \multirow[b]{2}{*}{ Grupos causas externas } & \multicolumn{2}{|c|}{1999} & \multicolumn{2}{|c|}{2000} & \multicolumn{2}{|c|}{2001} & \multicolumn{2}{|c|}{2002} & \multicolumn{2}{|c|}{2003} \\
\hline & $\%$ & Coef & $\%$ & Coef & $\%$ & Coef & $\%$ & Coef & $\%$ & Coef \\
\hline $\begin{array}{l}\text { Quedas e outras causas externas de } \\
\text { lesões acidentais }\end{array}$ & 37,05 & 35,33 & 35,83 & 24,06 & 35,08 & 28,00 & 34,37 & 27,46 & 35,84 & 33,16 \\
\hline Acidentes de transporte & 29,15 & 27,75 & 30,62 & 20,55 & 28,37 & 22,64 & 28,48 & 22,76 & 26,88 & 24,87 \\
\hline Suicídio* & 4,08 & 3,89 & 4,58 & 3,07 & 6,94 & 5,53 & 7,08 & 5,66 & 7,35 & 6,79 \\
\hline Homicídios** & 6,78 & 6,46 & 8,79 & 5,90 & 8,04 & 6,39 & 9,35 & 7,46 & 9,73 & 9,01 \\
\hline $\begin{array}{l}\text { Eventos cuja intenção é } \\
\text { indeterminada }\end{array}$ & 15,31 & 14,6 & 12,19 & 8,18 & 14,41 & 11,5 & 11,76 & 9,39 & 11,73 & 10,85 \\
\hline $\begin{array}{l}\text { Complicações da as sistência } \\
\text { médica e cirúrgica }\end{array}$ & 6,27 & 5,98 & 6,25 & 4,18 & 5,72 & 4,56 & 7,38 & 5,9 & 6,83 & 6,32 \\
\hline Sequelas de causas externas & 1,36 & 1,32 & 1,74 & 1,16 & 1,44 & 1,15 & 1,58 & 1,26 & 1,64 & 1,49 \\
\hline \multirow[t]{2}{*}{ Total } & 100 & 95,33 & 100 & 67,1 & 100 & 79,77 & 100 & 79,89 & 100 & 92,49 \\
\hline & \multicolumn{2}{|c|}{2004} & \multicolumn{2}{|c|}{2005} & \multicolumn{2}{|c|}{2006} & \multicolumn{2}{|c|}{2007} & \multicolumn{2}{|c|}{2008} \\
\hline Grupos causas externas & $\%$ & Coef & $\%$ & Coef & $\%$ & Coef & $\%$ & Coef & $\%$ & Coef \\
\hline $\begin{array}{l}\text { Quedas e outras causas externas de } \\
\text { lesões acidentais }\end{array}$ & 30,74 & 28,29 & 33,11 & 31,45 & 33,81 & 35,91 & 35,35 & 33,89 & 36,64 & 35,13 \\
\hline Acidentes de transporte & 31,32 & 28,82 & 29,88 & 28,38 & 30,21 & 32,08 & 28,08 & 26,91 & 28,21 & 26,95 \\
\hline Suicídio* & 5,64 & 5,19 & 7,36 & 6,99 & 5,97 & 6,34 & 6,56 & 6,29 & 6,09 & 5,83 \\
\hline Homicídios** & 9,77 & 8,97 & 10,32 & 9,71 & 7,66 & 8,12 & 8,44 & 8,08 & 7,06 & 6,77 \\
\hline $\begin{array}{l}\text { Eventos cuja intenção é } \\
\text { indeterminada }\end{array}$ & 14,05 & 12,93 & 12,84 & 12,19 & 19,54 & 20,76 & 19,09 & 18,3 & 19,2 & 18,4 \\
\hline $\begin{array}{l}\text { Complicações da as sistência } \\
\text { médica e cirúrgica }\end{array}$ & 7,63 & 7,02 & 5,96 & 5,66 & 2,58 & 2,74 & 2,22 & 2,13 & 2,68 & 2,56 \\
\hline Sequelas de causas externas & 0,85 & 0,76 & 0,56 & 0,52 & 0,24 & 0,22 & 0,26 & 0,24 & 0,15 & 0,14 \\
\hline Total & 100 & 91,98 & 100 & 94,9 & 100 & 106,2 & 100 & 95,84 & 100 & 95,78 \\
\hline
\end{tabular}

Fonte: Departamento de Informática do SUS (DATASUS), 1999 a 2008.

*CID10 - "Lesões autoprovocadas voluntariamente" 
A Tabela 3 apresenta a distribuição dos principais grupos de causas de óbito em idosos, entre as causas externas para o Estado de Minas Gerais. As quedas e demais causas externas de lesões acidentais representam a mais comum das causas externas de óbito em todos os anos analisados, seguindo-se dos acidentes de transporte. Existe uma flutuação discreta desses dois grupos de causas ao longo do período estudado, que respondem juntos a mais da metade das causas externas de óbitos em pessoas com 60 anos ou mais. Tomando-se os anos de 2000 e 2007 como referência para análises, registrou- se um aumento real do risco de morte em idosos por lesões acidentais da ordem de $40 \%$. Nesse grupo de causas incluemse as quedas, exposição a forças mecânicas, afogamento e submersão acidentais, riscos acidentais à respiração, exposição a corrente elétrica, fumaça, fogo e chamas, contato com fonte de calor e substâncias quentes, exposição a forças da natureza, envenenamento e intoxicação, entre outros. Nesse grupamento de causas, as quedas representam as principais causas. No mesmo período, o crescimento do risco de morte em idosos por acidentes de trânsito foi da ordem de $30 \%$.

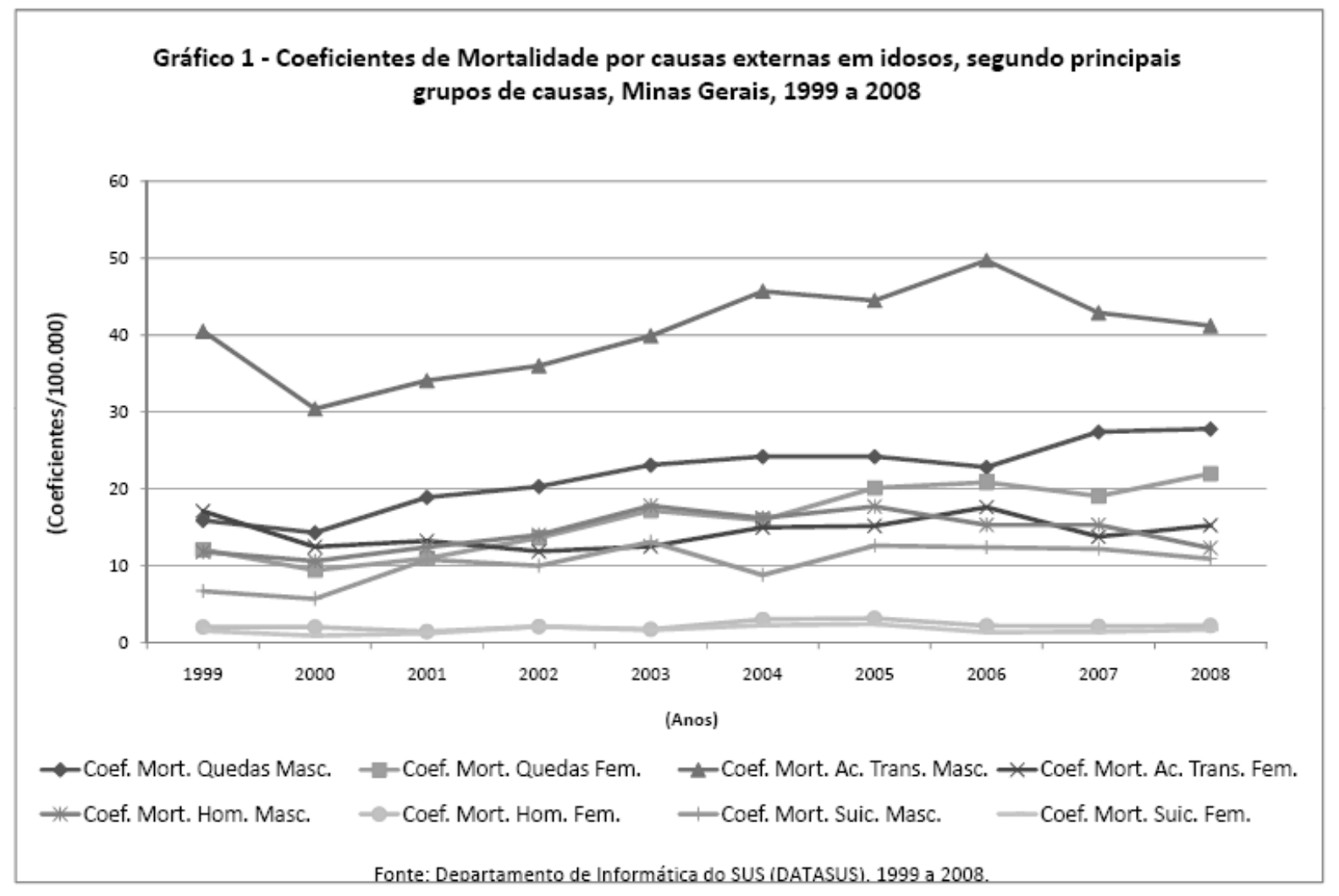

Uma análise gráfica dos principais grupos de causas segundo o sexo é apresentada no Gráfico 1. Os resultados mostram que a mortalidade por quedas aumentou ao longo da série estudada, passando de 14,3/100 mil em homens e 9,4/ 100 mil em mulheres em 2000 para 27,4/100 mil em homens e 19,1/100 mil em mulheres no ano de 2007. 0 risco de morrer em decorrência de quedas dobrou entre os idosos durante 0 período estudado. Ressalta-se que as quedas compreenderem intercorrências comuns entre os idosos, sendo causas de internações por resultarem em fraturas diversas, traumatismos cranianos e, eventualmente, levando ao óbito. 0 incremento do risco observado no período traz algumas indagações sobre as possíveis causas. Os primeiros questionamentos são sobre 0 acesso e a qualidade dos serviços de atenção à saúde do idoso. Muitas vezes, as quedas em idosos são decorrentes do uso de medicamentos inadequados. Outras vezes, o acesso a internações hospitalares é tardio e o surgimento de complicações decorrentes desse atraso culmina com a mor te do paciente. Sobre a frequência do evento 'queda' em idosos, um inquérito domiciliar realizado em Fortaleza mostrou que quase um terço dos idosos entrevistados passou por pelo menos um episódio de queda nos doze meses que antecederam a entrevista. Desse total, $20,3 \%$ relataram um episódio e $9,6 \%$, dois ou mais episódios. ${ }^{14}$ Tratando-se de um evento comum, é preciso, portanto, maior atenção para a população idosa no sentido de propiciar atendimento imediato e de qualidade e impedir evoluções desfavoráveis.

Outra particularidade do presente estudo foi a observação de que os coeficientes de mortalidade por quedas são maiores em pessoas do sexo masculino, enquanto, nos estudos realizados nas cidades de Maringá $(\mathrm{PR})^{4} \mathrm{e}$ Ribeirão Preto (SP) ${ }^{15}$, os coeficientes foram mais elevados para a população feminina. Deve-se considerar que taisestudos resultados mostram a realidade de uma única cidade, enquanto este estudo inclui o contingente de todo estado de Minas Gerais.

Em 2002, em um estudo realizado pela Organização Mundial de Saúde para avaliar o impacto mundial dos acidentes de transporte, constatou-se que a faixa etária de 60 anos e 
mais apresentaram as maiores taxas de mortalidade. Foram registradas cerca de 193.000 vítimas causadas pelo trânsito entre as pessoas nessa faixa etária. E, ainda, que a taxa de mortalidade foi a mais elevada de todos os grupos de idade para esses países ${ }^{16,17}$. No entanto, os acidentes de transporte aparecem neste estudo como a segunda causa de mortalidade.

Os coeficientes relativos aos acidentes de transportes podem ser considerados altos durante todo período, sendo em 2000, de 30,4/100 mil para homens e de 12,5/100 mil para mulheres e em 2007 de 42,9/100 mil para homens e de 13,8/ 100 mil para mulheres. Esses achados estão em consonância com outro estudo realizado nacionalmente, no qual se registraram os óbitos da população geral no período de 1977 a 2004 com apontamentos de elevados coeficientes de mortes por acidentes de transporte. ${ }^{7}$

Acrescenta-se ainda que, no último ano, a mortalidade para o homem idoso foi maior nos casos de acidentes de transporte (41,2/100 mil), quedas (27,8/100 mil), homicídios (12,3/100 mil) e suicídios (10,9/100 mil). Para as mulheres, os coeficientes de mortalidade foram maiores para as quedas (22,0/100 mil), seguido dos acidentes de transporte (15,2/ $100 \mathrm{mil})$.

Alguns autores discutem a distribuição dos óbitos na sociedade moderna, e Minayo ${ }^{8}$ pondera que 0 aumento dos casos de morte por homicídio e acidentes de trânsito entre homens pode estar vinculado ao estilo de vida moderno, às desigualdades crescentes na sociedade, à impunidade das infrações e delinqüências e à exclusão social, dentre outras causas. A partir dos dados apontados por este estudo, questiona-se se o estilo de vida adotado por adultos jovens não estaria alcançando os idosos. Todavia, torna-se necessário o desenvolvimento de estudos acerca da vulnerabilidade dos idosos, principalmente homens, em relação aos acidentes de transporte e homicídios.

Sobre os homicídios em 2008, foram identificados 145 mortes decorrentes de agressões em Minas Gerais entre idosos, correspondendo a 7,06\% do total de óbitos por causas externas. Os coeficientes encontrados foram de 12,3/100 mil homens e 2,2/100 mil mulheres. Em relação à população geral, em um estudo realizado no Brasil, no período de 1977 a 19947, observou-se que o percentual encontrado é quase três vezes menor, pois o referido estudo acusa que os homicídios foram responsáveis por $18,08 \%$ do total de mortes por causas externas. Portanto, a participação de causas violentas entre os óbitos em idosos parece ser maior em outras unidades federativas.

Os suicídios apresentaram coeficientes em torno de 5,7/100 mil homens e 0,9/100 mil mulheres em 2000 e passaram para 12,2/100 mil homens e 1,4/100 mil mulheres em 2007. É curioso observar que, enquanto a série estudada não apresentou significativas alterações para esse grupo de causas entre as mulheres, apontou, por outro lado, a duplicação do risco para os homens. Apesar desse incremento, a
Organização Mundial de Saúde ${ }^{18}$ registra que esses valores podem ser considerados baixos quando comparados com países da Europa, que possuem coeficientes acima de 20/100 mil habitantes para a população geral.

Discutindo sobre o suicídio como causa de óbito, Melo e colaboradores ${ }^{7}$ afirmam que os coeficientes de mortalidade por suicídio são sempre maiores no sexo masculino, contrariamente às tentativas mais numerosas entre as mulheres do que entre os homens. Além disso, apresentam taxas mais elevadas em populações mais idosas, como mostrado neste estudo, ocorrendo, entretanto, desde a adolescência. No entanto, os dados do Sistema de Informação sobre Mortalidade mostram que o risco de morrer por suicídio ainda é maior em adultos jovens - faixa etária de 20 a 39 anos - com o coeficiente de mortalidade de 5,47/100 mil em 2000 e 6,96/100 mil no ano de 2007. Ressalta-se que a mortalidade por suicídios de idosos no ano de 2007 foi bem próximo ao de adultos jovens. As razões para essas diferenças implicam novos estudos.

Em relação aos óbitos por eventos nos quais a intenção é indeterminada, verificou-se uma flutuação dos valores ao longo dos anos estudados (Tabela 3). É impor tante ressaltar que esse coeficiente traduz a proporção de causas indeterminadas, e, portanto, seria desejável sua redução ao longo do período estudado. 0 esclarecimento dessas causas é muito importante, pois a mortalidade por agressões e autoprovocada pode estar incluída nessa categoria². Nesse contexto, o crescimento da mortalidade por eventos de intenção indeterminada no decorrer dos anos pode ser decorrente de uma qualidade precária sobre as informações de mortalidade para o Estado de Minas Gerais. Resultados distintos foram encontrados no estado do Paraná no qual houve uma redução de 22,3\% em 1980 para 4,6\% em 1999, indicando a melhoria da qualidade das informações sobre as circunstâncias do óbito. ${ }^{2}$

\section{CONSIDERAÇÕES FINAIS}

Este estudo possibilitou descrever as características da população idosa em relação à mortalidade por causas externas. Neste trabalho, evidenciou-se o crescimento das causas externas entre os idosos em Minas Gerias, no período de 1999 a 2008, por meio dos coeficientes de mortalidade.

A partir dos coeficientes de mortalidade para idosos na série histórica estudada, verificou-se que o risco de morrer se elevou no período intercensitário. Mesmo compreendendose a complexidade de fatores envolvidos com as causas externas de mortalidade, é preciso questionar o impacto das medidas governamentais diante do crescimento observado.

A mortalidade pelos acidentes de qualquer natureza e decorrentes da violência social na população idosa mineira é um fato inquestionável e que requer intervenção urgente. Ressalta-se que, inicialmente, é necessário o conhecimento do problema. No entanto, o conhecimento epidemiológico sozinho do problema não resolve nenhuma situação, pois se trata de 
um fenômeno complexo. Medidas de cuidados e proteção assumidas desde os níveis primários de atenção à saúde poderiam reduzir os coeficientes de mortalidade em idosos, como, por exemplo, na abordagem das quedas neste grupo etário. Tal evento (óbitos por quedas) praticamente duplicou no período estudado. 0 conhecimento do ambiente onde o idoso vive, bem como a adaptação de alguns lugares da residência, deve ser o ponto de partida no sentido de se reduzir barreiras e evitar as quedas. A educação em saúde com idosos e cuidadores deve ser assumida como forma de definir corresponsabilidades sobre possíveis eventos decorrentes do processo de envelhecimento e treinamento para intervenções preventivas e para um bom cuidado dos idosos.

Foram constatados coeficientes de mortalidade por acidentes de transporte na população idosa em toda a série. Esse fato deve ser conhecido pelas autoridades competentes e por todos aqueles responsáveis pelas ações de planejamento de educação no trânsito, objetivando o estabelecimento de políticas públicas para minimizar essas mortes.

Também a mortalidade por homicídios e suicídios entre homens idosos destaca a necessidade de ações específicas, pois apresentou crescimento significativo no período estudado. Recomenda-se o desenvolvimento de novos estudos para melhor delinear a vulnerabilidade, principalmente do homem idoso em relação aos acidentes e violências.

Finalmente registrou-se uma qualidade precária das informações registradas no banco de dados do Sistema de Informação sobre Mortalidade (SIM) para o grupo populacional de 60 anos ou mais, evidenciada pelo aumento de óbitos por eventos cujas causas são indeterminadas. Com isso, muitas causas de óbitos ficam subnotificadas, o que prejudica o amplo conhecimento do problema para o planejamento e prevenção em saúde. Torna-se necessária a capacitação dos técnicos e codificadores e a sensibilização das equipes médicas, que são responsáveis pelo preenchimento das declarações de óbito, para que o documento seja tomado como um instrumento de geração de dados, e não como mera formalidade do processo de sepultamento.

\section{REFERÊNCIAS}

1.Minayo MCS, Souza ER, organizadores. Violência sob o olhar da saúde: a infrapolítica da contemporaneidade brasileira. Rio de Janeiro (RJ): Ed Fiocruz; 2003.

2.Lozada EMK, Mathias TAF,AndradeSM,Aidar T. Informações sobremortalidade por causas externas e eventos de intenção indeterminada, Paraná, Brasil, 1979 a 2005. Cad. Saude Publica [periodico na Internet]. 2009 jan [citado 2010 abr 05]; 25(1): 223-228. Disponivel em: http://wnw.scielosp.org/ scielophp?script=sci_arttext\&pid=S0102311 X2009000100024\&lng=en . doi: 10.1590/50102-311X2009000100024.
3.Mello Jorge MPH, Latorre MRDO. Acidentes de trânsito no Brasil: dados e tendências. Cad Saude Publica 1994; 10(supl 1):19-44.

4.Mathias TAF, Jorge MHPM, Andrade OG. Morbimortalidade por causas externas na população idosa residente em município da região sul do Brasil. Rev. Latino-am. Enfermagem[periodico na Internet]. $2006 \mathrm{fev}$ [citado 2010 abr 05]; 14(1): 17-24. Disponível em: http://www.scielo.br/ scielo.php?script=sci_arttext\&pid=S010411692006000100003 \&lng =en.doi:10.1590/S010411692006000100003

5.Gawryszewski VP, Jorge MHPM, Koizumi MS. Mortes e internações por causas externas entre os idosos no Brasil: 0 desafio de integrar a saúde coletiva e atenção individual. Rev Assoc Med Bras [periodico na Internet]. 2004 [citado 2010 abr 05]; 50(1): 97-103. Disponivel em: http://www.scielo.br/ scielo.php?script=sci_arttext\&pid=S010442302004000100044\&lng=en. doi: 10.1590/S0104-42302004000100044.

6.Instituto Brasileiro de Geografia e Estatística- IBGE. Censo 2000 [acesso 2010 mar 29]. Disponível em: http://www.ibge.gov.br/home

7.Mello Jorge MHP, Gawryszewski VP, Latorre MRDO. I: análise dos dados de mortalidade. Rev Saude Publica 1997; 31 (supl 4): 5-25.

8.Minayo MCS, Souza ER. É possível prevenir a violência? Reflexões a partir do campo da saúde pública. Cienc Saude Colet 1999; 4(1): 7-32.

9.Minayo MCS. The inclusion of violence in the health agenda: historical trajectory. Cienc Saude Colet 2006; 11: 375-83.

10.Aidar T. 0 impacto das causas violentas no perfil de mortalidade da população residente no município de Campinas: 1980 a 2000. Rev Bras Estud Popul 2003; 20: 281-302.

11. Ministério da Saúde (BR). Estatuto do Idoso. Brasilia (DF); 2003 [acesso 2010 jul 27]. Disponível em: http://bvsms.saude.gov.br/bvs/ publicacoes/estatuto_idoso.pdf.

12. Ministério da Saúde (BR). DATASUS. [base de dados na Internet]. Informações de saúde demográficas e socioeconômicas. Projeções intercensitárias Brasilia(DF); 2005 [citado 2010 mar 12]. Disponível em: http://tabnet.datasus.gov.br.

13.Organização Mundial da Saúde- OMS. Centro Brasileiro de Classificação de Doenças em Português. Classificação Estatística Internacional de Doenças e Problemas Relacionados à Saúde. 10ª rev. São Paulo (SP): EDUSP; 1998.

14. Coelho Filho JM, Ramos LR. Epidemiologia do envelhecimento no Nordeste do Brasil: resultados de inquérito domiciliar. Rev Saude Publica 1999 out; 33(5): 445-53.

15.Fabrício SCC, Rodrigues RAP, Costa Junior ML. Causas e conseqüências de quedas de idosos atendidos em hospital público. Rev Saude Publica 2004 fev; 38(1): 939.

16.Abreu AMM, Lima JMB, Griep RH. Acidentes de trânsito e a frequência dos exames de alcoolemia com vítimas fatais na cidade do Rio de Janeiro. Esc Anna Nery . 2009 jan/mar; 13 (1) 
17. Organización Mundial de la Salud-OMS. Informe mundial sobre prevención de los traumatismos causados por el transito. Resumen. Ginebra; 2004.

18. Organización Mundial de la Salud-OMS. World health statistics annual 1993. Geneva; 1994. 